\title{
Epigenetics of Metabolic Syndrome as a Mood Disorder
}

\author{
Sermin Kesebir
}

\begin{abstract}
Mood disorders comprise major depressive disorder (MDD), bipolar disorder (BD) and the milder forms of these two disorders. Reccurring MDD is also known as unipolar disorder. The distinction between unipolar and bipolar disorders was first suggested in 1957 by Leonard and was made official after support by several studies in 1980. Indeed, in 150 AD, Aretaeus of Cappadocia wrote "It seems to me that melancholia is the beginning and a part of mania". Additionally, Kraepelin, who proposed the first medical disease model in psychiatry a century ago, considered recurrent unipolar depression cases under the category of bipolar disorder and conceptualized spectrum disorders. Because today's classification systems conduct crosssectional diagnosis, they do not consider family history, long-term characteristics and multidimensional approaches on symptoms. This method prioritizes reliability over validity and it rules out psychiatric disorders in etiology. Actually, a spectrum model which covers physical diseases is conceivable. The concept of epigenetics considers mood disorders, Alzheimer's disease, attention deficit and hyperactivity disorder, Carney syndrome, multiple endocrine neoplasia type I and II, breast and prostate cancers, carsinoid tumors, cerebrovascular and cardiovascular diseases and metabolic syndrome together. This review addressed the relationship between metabolic syndrome and mood disorders in this context along with genetic, clinical and environmental factors such as climate, geographic factors, migration and changeable lifestyles. Genetic and clinical variables are affective temperament, childhood trauma and use of antidepressants and antipsychotics.
\end{abstract}

Keywords: Metabolic syndrome; Mood disorder; Temperament; Childhood trauma; Drugs; Climate; Geographic factors; Migration

\section{Introduction}

It is known that bipolar disorder (BD) is associated with premature mortality [1]. Excessive mortality rates caused by medical disorders are around 1.5 - 3 times higher in adults with $\mathrm{BD}$ in comparison to the population in general. There is increasingly more abundant evidence that shows a relationship between

Manuscript submitted February 18, 2018, accepted March 26, 2018

Uskudar University, NPIstanbul Brain Hospital, Ahmet Tevfik Ileri C. N: 18, 34768 Umraniye, Istanbul, Turkey. Email: serminkesebir@hotmail.com

doi: https://doi.org/10.14740/jocmr3389w mood disorders and some physical diseases including obesity (waist circumference), hypertension, diabetes, dyslipidemia, cardiovascular and cerebrovascular disease [2]. Intersections indicating pathophysiological relationships between BD and general medical conditions that are susceptible to stress such as metabolic syndrome (MetS) include glucocorticoid/insulin signaling mechanisms and inflammatory effector systems.

\section{MetS and BD}

MetS is found more frequently in patients of BD than it is in the general population [3]. A patient subgroup with BD has a higher probability of developing MetS according to their habits, lifestyles, genetic susceptibility, and preference of treatment. Studies reported a prevalence of $35-50 \%$ for MetS in BD patients, and MetS was associated with obesity, hypertension, diabetes and dyslipidemia. While these are not the diagnostic criteria for MetS, the framework of MetS is considered to include pro-inflammatory and prothrombotic states and purinergic dysfunction $[4,5]$.

Patients of BD with MetS have an adverse course and outcome, more unfavorable response to treatment, a higher probability of suicidality, higher unemployment and thus higher level of financial issues. Meanwhile, having a medical condition was related to longer duration of untreated illness and being female [6]. Perugi et al associated the length of pharmacological treatment and age at onset of first major episode with MetS comorbidity [7].

Specific MetS characteristics in psychiatric population usually include early age of onset, hyperinsulinemia, higher abdominal adiposity, and lower levels of high-density lipoprotein (HDL) cholesterol, whereas the common denominator of these may be insulin-resistance [8]. Our study compared patients with or without MetS, and the mean age of MetS (+) patients was lower in the first manic episode (FME) with previous depressive episode (PDE) group, while it was higher in FME without PDE group [9]. To clarify, onset of BD was earlier in MetS (+) patients in the FME with PDE group. However, the MetS $(+)$ patients in the FME without PDE group were older. Therefore, the onset of BD was comparatively later in patients with MetS in this group. In compliance with this result, a previous study of ours where we investigated the incidence of diabetes in first episode mania patients found late onset to be one of the predictors of diabetes development [10]. Additionally, in a more recent study of ours where we studied cellular adhesion molecules as a component of pro-inflammatory processes in first episode mania without previous depressive episode, the onset age was found as $30.5 \pm 9.9$ and this result may be con- 
sidered as relatively old for BD onset [10].

It is a vital but yet unanswered question whether or not there is a connection of time or phase between BD and MetS, because both disorders have negative effects on the prognosis of each other and on selection and implementation of treatment modalities for both. Soreca et al proposed that comorbidity of medical conditions was independent of age but associated with duration of $\mathrm{BD}$ and they were related to some mechanisms and biological determinants that are common [11]. In one of our prospective studies where we evaluated 2,000 consecutive patients who were admitted to our outpatient clinic and provided informed consent, we examined the process of diagnosis and treatment of MetS according to NCEP ATP III criteria in patients who were diagnosed with schizophrenia, $\mathrm{BD}$, recurrent major depressive disorder (MDD) and anxiety disorder (generalized anxiety disorder, panic disorder, and obsessive-compulsive disorder) based on the DSM-IV criteria [12]. The study mentioned here, assessments of 1,816 patients were found to be reliable and covered in statistical analysis. When we tested the correlations of the time periods since onset of axis I psychiatric disorder and onset of MetS, although these durations were found similar in the affective disorders group $(6.19 \pm 7.55$ and $7.12 \pm 8.15)(r=0.912)$, the value of $\mathrm{r}$ was 0.265 for the anxiety disorders group $(3.21 \pm 3.15$ and $8.34 \pm 5.71)$ and 0.425 for the schizophrenia group (13.82 \pm 11.36 and $8.21 \pm 8.55$ ). These results mean that the times of onset for affective disorders and medical conditions were relatively concurrent.

When comorbid medical conditions were evaluated based on phases of $\mathrm{BD}$, they are possibly more prevalent on onset and in earlier episodes. This is because early mortality is found more among patients with earlier onset [13]. Medical condition comorbidities that rise in the middle stages of BD could potentially be associated with the influence of treatment and effects of patient's habits and lifestyle. On the other hand, it was demonstrated that even in these circumstances, they emerge one decade earlier than the patients without BD at the same ages. Considering these findings together, it appears that MetS is one of the parameters that are initiators and outcomes of BD.

\section{MetS and Psychotropic Drugs}

In our study which examined MetS in patients with FME which accounted for the presence of a previous depressive episode, MetS was found to be more prevalent in the FME with PDE group in comparison to FME without PDE [13]. Furthermore, PDE presence was found to be the strongest predictor of MetS in regression analysis. Clearly, individuals with depression have a higher risk of MetS [14]. Likewise, according to our results, four-fifths of the patients with PDE had received psychopharmacological treatment for their stated depressive disorders [9]. Consequently, it is worth asking whether depressive episode itself or the psychopharmacological agents used for treatment of it was the cause of higher MetS among FEM patients with PDE.

Vancampfort et al's meta-analysis stated that usage of antipsychotics explained higher MetS prevalence estimates in MDD significantly. Differences in MetS prevalence were not mediated by age, sex, geographical area, smoking, antidepressant use, and presence of psychiatric comorbidity. Another study found some mediation role for tricyclic and nonselective serotonin-reuptake inhibitor antidepressant use, but overall, clinical differences' mediating roles were limited [14]. Margary et al evaluated 83 psychiatric inpatients diagnosed with schizophrenia, BD and MDD, and they showed a positive relationship between antidepressant drug treatment with triglycerides, and triglycerides/HDL ratio levels and antipsychotics drugs with the HOMA and Framingham indices [15]. Perugi et al associated duration of pharmacological treatment and age at onset of first major episode with MetS comorbidity [7].

There are conflicting results that there may or may not be a relationship between usage of tricyclic antidepressant and incidence of pheochromocytoma and adrenal gland tumors [16, 17]. In addition to psychological stress, it is an important question what role noradrenaline plays in physical diseases besides hypertension. Epidemiologic studies should test whether this risk is decreased by drugs that reduce the level of noradrenaline or make noradrenergic receptor blockade.

\section{The Mediator Role of Childhood Trauma}

Our study associated negative family history of BD with MetS for the first time [3]. It was proposed as one of the predictors of MetS in patients with first episode mania. This result is highly significant as it proposes potential alternative etiological links apart from MetS and genetic factors for BD whose monozygotic concordance is $70 \%$. It was shown in molecular genetic studies that, BD shares similar conversions and deletions in the same loci as some medical conditions in general including coronary artery disease, hypertension, diabetes mellitus type I and II [18]. On the other hand, only $10 \%$ of the total variance of clinical co-existence may be explained by genetic association [19]. This outcome, called "missing heritability" by researchers, means that interactions with environmental effects have an absolute role both in etiology and resilience in accordance with epigenetic principles [20]. Our study found childhood trauma to be another predictor of MetS [3]. The relationship mentioned here was also suggested earlier by McIntry et al [21].

Acute stress induces a response in the brain by an inflammatory reaction [12]. Autonomic nervous system is activated directly. Release of adrenaline and noradrenaline results in binding of these to alpha and beta-adrenergic receptors on cytokine cells. Then, nuclear factor kappa-beta-mediated proinflammatory cytokine release begins. Meanwhile, chronical stress causes HPA axis disorders and consequent hypercortisolism. Thus, childhood traumas are frequently linked with obesity, diabetes, coronary artery disease, chronic obstructive pulmonary disease and autoimmune diseases. At this time, abnormal stress response could act in the etiology of both a chronic psychiatric disorder and an accompanying medical condition. Medical conditions linked with childhood trauma in BD include hypertension and obesity [22]. Moreover, early menarche and EEG abnormalities are found as the projections 
of childhood trauma on $\mathrm{BD}[23,24]$.

\section{Mediator Role of Environmental Factors Such as Climate, Geographic Factors, Migration and Changeable Lifestyles}

Despite the clearer demonstration of its genetic aspects in recent years, seasonality is a variable which may also be assessed in the context of epigenetic principles, and per our results, it is a predicting clinical factor for MetS in first episode manic cases. Environmental factors including seasonality affect vulnerability to allostatic load. It is amply documented that bipolar symptoms or episodes are affected by seasonality in susceptible subsets. It may be considered that MetS is a phenotypic indicator of an abnormal stress response with somatic manifestations [22]. It would be worth knowing whether individuals with MetS seasonality are more or less likely to additionally experience breakthrough symptomatology. Seasonal variations in behaviors are also generated by the principal circadian clock. Seasonality increases the risk for MetS, and evidence shows that disruption of the clockwork may cause changes in metabolism. Englund et al supported the relationship between circadian clocks and MetS [25]. Circadian gene variants were associated with the risk factors of MetS, like they were associated with hypertension and high fasting blood glucose.

MetS is associated with high global scores on seasonal changes in mood and behavior, and with particularly those in weight. Evaluation of these alterations may be a useful indicator of MetS due to easy assessment. Our study found MetS to be more prevalent in the winter type of seasonal affective disorder (SAD) [26]. The winter and summer types of SAD are different from each other in terms of prevalence of MetS. Actually, abnormalities in the circadian clockwork which links seasonal fluctuations to metabolic cycles may prompt seasonal changes in weight, and consequently, MetS. Meanwhile, as reported by some studies, circadian symptoms of depression induce such metabolic processes in the body, which eventually leads to obesity and insulin resistance [27]. Our study found eating attitude trait (EAT) scores to be similar between subjects with winter and summer types of SAD, higher in METS $(+)$ SAD subjects than MetS (-) SAD subjects and higher in MetS (+) winter type of SAD subjects than MetS (+) summer type of SAD subjects [26]. Studies have associated higher EAT scores with atypical depression.

A systematic study assessed 8,028 individuals who took a nationwide health examination survey in Finland using the ATPIII criteria for MetS and with SPAQ regarding seasonal changes in mood and behavior [28]. Particularly seasonal changes in weight were a risk factor for MetS, after controlling for a number of known risk and potential confounding factors. The probability of having MetS rose by $56 \%$ among those with a global score equal to winter blues or more severe seasonal changes in their mood and behavior. It was reported that disruptions in the circadian clockwork are linked with and may predispose MetS.

In our sample, the summer type of SAD was more frequent $(56.8 \%)$, whereas MetS was found to be more frequent $(94.1 \%$ vs. $64.3 \%)$ in the winter type of SAD [26]. As expect- ed, prevalence of obesity as defined by the World Health Organization (WHO) was relatively low in Asia in comparison to western countries [29]. Thus, Han et al stated that, as opposed to the case in western countries, the summer type of SAD was more prevalent than the winter type of SAD in China [30]. Levitan reported in 2007 that SAD was independent of latitude differences [31]. In the study in Turkey on SAD with the largest sample, Elbi et al screened 3,229 participants with SPAQ in eight different regions and found no correlation between latitude and the prevalance of the disorder [32]. Tonetti et al compared Italy and India [33]. They found that, while the winter type of SAD was more prevalent in Italy, the summer type of SAD was more prevalent in India. As they thought the first result was associated with reduced duration and intensity of light in winter, in other words with photoperiodicity, they suggested the second result was associated with climatic and thermal differences. Guzman et al compared African migrant and Afroamerican subjects living in Washington, and observed that while the winter type of SAD was similar in the two groups, the summer type of SAD was more prevalent among African migrants [34]. Comparative studies showed that metabolic reactions to obesity may be higher in South and East Asians than their western counterparts at given body mass indices (BMI) [29]. The climatic, ethnic, geographical, genetic and cultural interactions of SAD appear to be worth researching over the next years.

No patients with the summer type of SAD were found in Iceland and England [35]. Although Iceland is far towards the north, MDD prevalence was not higher than England. This may be understood both as an outcome of their tolerance to photoperiodicity due to isolation for more than a thousand years, and as an outcome of genetic factors. Indeed, SAD is seen less frequently among migrant Icelanders than among other ethnic migrant groups. Authors also underlined the significance of weather conditions and living in rural or urban areas. In fact, living in a rural area means exposure to more light than the other parts of the region. In our study, all of the patients of the summer type of SAD were living in urban areas. As an interesting but not surprising result, all MetS (+) patients were living in cities as well.

Grimaldi et al tested which environmental, social, lifestyle and health-related factors of people contributed to seasonal differences in moods and behaviors and whether these factors influenced the possibilities of MetS and MDD, both of which are conditions with high prevalence among industrialized populations. A total of 5,480 individuals aged 30 and older in Finland who were representative of the general population were examined for MetS using the ATP-III criteria, they provided self-reported information on seasonal variations in mood and behavior, and the DSM-IV criteria were used to interview them for mood, anxiety, and alcohol use disorders [36]. Seasonal variations in mood and behavior have a metabolic factor comprising weight and appetite, and greater loadings on this factor increase the risk of MetS. Self-reported information on lighting assessed to be poor at home contributed to scores for the metabolic factor.

Comorbidity was more prevalent in the winter type of SAD and in MetS (+) patients than MetS (-) patients [27]. The comorbid diagnoses included anxiety disorders and alcohol 
and substance use disorders. When MetS $(+)$ winter type and summer type SAD patients were compared, anxiety disorders were more prevalent among MetS $(+)$ winter type SAD patients whereas alcohol and substance use disorders were more prevalent among MetS (+) summer type SAD patients. The results studies with migrants on SAD provided quite interesting data. A study was conducted among five different migrant groups living in Oslo, and it was found that the winter type of SAD was the least prevalent among Sri Lankan migrants and the most prevalent among Iranian migrants [37]. The winter type of SAD was linked to place of birth, young age, childhood trauma, frequent internal medicine and psychiatry referrals, while the summer type of SAD was linked to place of birth, smoking and alcohol consumption. Their results were in parallel with our findings. Our findings here extend the seasonal pattern to the seasonal variations in mood and behavior that are part of not only depression but also anxiety and alcohol use disorders.

Additionally, there is a chance for seasonal changes in mood and behavior to run in families, especially seasonality of the winter type, and this is mostly caused by a biological tendency [38]. In our study, presence of family history was more prevalent in patients with the winter type of SAD [27]. On the other hand, this difference ceased to exist when we compared the MetS (+) summer and winter type of SAD groups. Additionally, when we compared MetS (+) and (-) SAD patients, we did not observe any difference in terms of family history. Once again, this result highlights the contribution of environmental factors on MetS observed in SAD.

Due to the changes in the information and feedback received, changing seasons challenge the circadian clock functions. Changing seasons may challenge a switch between the metabolic and circadian-based actions of time-keeping mechanisms. Light-dark transitions are necessary for resetting the master circadian clock on a daily basis. In cases where these signals are missed, as it occurs with the decreasing photoperiod in general and the shortage of light exposure in morning hours during winter in particular, the circadian clock tends to get delayed and the circadian clockwork may start relying more on metabolic cycles producing time feedback required for adaptation [39]. The circadian clockwork and its integrity are challenged by the periods of fall and spring the most. Research on animals showed that, in spring, within the master circadian clock, the morning-tagged or morning-active cells yield the dominance to the evening-active cells, or from waking up to sleep onset process [40]. This may require input from the intrinsic clock which reacts to a change in seasons and runs its targets [41].

In people with seasonal variations in their mood and behavior, physical activities usually decrease, which alters the effect of exercise on and the feedback from a peripheral circadian clock of the skeletal muscle to the master circadian clock [42]. This may lead to delays in the circadian clockwork. Moreover, craving carbohydrates in the evening is a frequently seen sign which may lead to delays in the circadian clockwork [43]. In seasonal affective disorder patient, there are also increases in the resting metabolic rate during a depressive episode in winter [44]. The action mechanisms here are still illuminated at a molecular level concerning a conceivable network or a potential pathway that might link the light-exposure and food-intake responsive oscillators to a variety of affective and behavioral outputs [40]. Lighting conditions and their dynamics may be a measure for involvement to affect the seasonal metabolic signals and in the end to prevent MetS.

Indeed, a subtype of depressive episode (melancholic subtype), number of manic episodes and seasonality were distinguished in lithium-induced hyperparathyroidism before lithium use [45]. 25(OH) vitamin D levels were lower and calcium and parathyroid hormone (PTH) levels were higher in bipolar patients with attention deficit hyperactivity disorder (ADHD) comorbidity [46]. 25(OH) vitamin D status is related to behavioral and affective disorders; however, pathophysiological mechanisms may be different in two disorders [47]. It would be better to examine both $25(\mathrm{OH})$ vitamin D levels and serum PTH levels in bipolar patients with ADHD comorbidity.

Total sleep time and sleep latency were found to be longer in the winter type of SAD in comparison to the summer type of SAD [27]. There was similarity between the durations of delayed phases of the patients of the winter and summer type of SAD. When MetS (+) and (-) SAD patients were compared, sleep latency was longer, whereas total sleep time was shorter in the MetS (+) SAD group. Moreover, the duration of delayed phase was found to be significantly longer in MetS (+) patients. There was no difference between MetS $(+)$ summer and winter type SAD patients in terms of delayed phase duration. Total sleep time was similar between the two groups, whereas latency was longer in summer type SAD patients.

Delayed sleep phase syndrome (DSPS) and SAD may manifest similar delayed circadian phase issues [48]. Signs of DSPS were $26 \%$ in SAD and $2 \%$ in controls. Shorter sleep length and longer sleep-onset latency were reported by the patients.

In agreement with our results, the study referenced above reported that, longer sleep showed lower chances of signs of MetS. DSPS or namely a dysfunctional circadian system may be a mechanism that compensates for degraded cellular-level energy production [49]. Therefore, mitochondrial calciuminduced oxidative phosphorylation. Higher calcium levels observed in some cases of mania could be a resource for the higher levels of mitochondrial respiration also observed in depression. Here, we would like to remind that calcium levels affect the activity of the circadian clock and levels of circadian clock gene outputs. Circadian activity governed a firmly self-regulated oscillatory rhythm in the manifestation of the circadian-controlled gene. However, circadian regulation of interconnected translation transcriptional feedback loops was verified to be misleading. As this is the way it is, fidelity and plasticity of the circadian clock are maintained by post-translational modification of clocks proteins, the behavior of certain microRNAs and cyclically coordinated epigenetic control of clock protein transcription [50].

\section{Mediator Role of Affective Temperament Be- tween Childhood Trauma and Environmental Factors in MetS and/or BD}

Temperament begins in the brain structure, and hereditary individual differences are attributable to varieties in neural and physiological functions [51]. Temperament was suggested to 
be associated with MetS signs, which may be partly mediated by lifestyle and socioeconomic status. It was proposed by Altinbas et al that depressive temperament profiles may incline an individual to the development of MetS in the winter [52]. They found the proportions of MetS as $19.2 \%, 23.1 \%, 34.6 \%$ and $38.5 \%$ in the summer, fall, spring, and winter, respectively. Only depressive temperament scores were reported to be higher in patients with MetS during the winter.

Neuroticism and openness were established as aspects related to seasonal mood changeability [53]. Furthermore, the study revealed a relationship between predisposition to mild winter depression and an avoidance-oriented coping style. The avoidance coping style was correlated positively with all aspects of seasonality described by SPAQ (correlation coefficients ranged from 0.21 to 0.34 ). Both sub-types of avoidanceoriented style, namely distraction and social diversion, were linked to marked subjective seasonal changes in sleep length, mood and levels of energy. It was proven that the subjective amplitude of circadian rhythm is associated with seasonality, whereas the subjective acrophase of the rhythm (morningnesseveningness preference) did not.

Over a 3-year period, temperamental factors were associated cross-sectionally with, as well as predicted for, the MetS precursors [54]. Mental vitality was found as likely to be linked with positive emotionality and positive emotionality was found as likely to be linked with a low MetS risk level, whereas hyperactivity, negative emotionality, responsivity to others, and cooperativeness were related to a high level of MetS risk. The same researchers' findings demonstrated that a temperament profile characterized by a high level of perseverance and reward dependence, an average level of novelty seeking, and a low level of harm avoidance were linked with a high level of MetS risk factors [55]. A systematic review on 13 cross-sectional analyses and 10 longitudinal analyses associated hostility, anger, type A behavior and neuroticism and type $\mathrm{D}$ personality with an increased frequency of MetS and its development over time [56]. Our study differentiated two types of affective temperament between MetS (+) and (-) subjects: anxious and irritable temperaments [3]. Hyperactivity, high level of persistence and reward dependence, average level of novelty seeking, and low level of harm avoidance which were described in previous studies are similar to the characteristics defined for irritable temperament. Moreover, negative emotionality, responsivity to others and cooperativeness are aspects in line with the aspects defined for anxious temperament. In terms of responsivity to others and cooperativeness, anxious temperament overlaps with depressive temperament. In this context, our findings are also consistent with Altinbas et al's results [52].

Some studies also emphasized the role of sex in the connection between temperament and MetS. Ravaja et al reported that the link between MetS and hyperactivity and negative emotionality was more prominent in men [54]. Sovio et al assessed the relationship between temperament and MetS in Northern Finland 1966 Birth Cohort [57]. Accordingly, it was found that novelty seeking was positively related to waist circumference in both sexes. However, systolic blood pressure was the highest in men with high harm avoidance and low persistence scores, a profile consistent with anxious temperament and the lowest in women with high reward dependence and high per- sistence scores, a profile consistent with irritable temperament. In a previous study of ours, we observed a relationship between impulsivity and triglyceridemia specific for anxious temperament [58]. When it is assessed separately for sexes, the association between impulsivity and triglyceride levels was observed only in female bipolar patients [59]. In a later study of ours following the aforementioned one where we investigated MetS components by sex in first episode subjects, we observed that the triglyceride levels of female patients were different from healthy controls [60]. In our last study, sex difference between MetS (+) and (-) patients was close to statistical significance only in the FME with PDE group, and it was more prominent in females [9]. It is known that anxious temperament is more common among men and irritable temperament is more common among women [51]. Anxious temperament differentiated in favor of MetS (+) in the FME with PDE group whereas the female sex was dominant. Moreover, irritable temperament differentiated in favor of MetS as well in the FME without PDE group whereas sex distribution was similar between MetS (+) and (-) patients. We believe the reason for irritable temperament scores' invariability in favor of MetS in the FME with PDE group could be the predominantly female sex distribution in this group. Being female is a risk factor for MetS [6]. Our results suggested that the same applies to FME with PDE [9].

Affective temperament is also a proposed endophenotype for BD. Temperaments found to be genetically linked with BD are cyclothymic and hyperthymic temperaments, and there are different in patients and their healthy counterparts among controls [51]. In our study, anxious and irritable temperaments which differentiate in MetS (+) patients appeared to underline the effects of environmental conditions on development of MetS again [19]. Irritable temperament was linked to mixed episodes in patients with $\mathrm{BD}$ [61]. McIntyre reported that obesity may influence the symptomatic manifestation of BD by increasing the probability that these patients will manifest mixed episodes [21]. We believe this is applicable to not only obesity but also MetS. Unsuitable usage of psycopharmacological antidepressants may contribute to this condition directly by raising the risk of having mixed episodes and indirectly by raising the risk of MetS.

In contrast, there was no clear link between temperament measures and the occurrence and development of MetS. Our last study found triglyceride levels to be correlated with hyperthymic, irritable and anxious temperament scores [62]. HDL levels and irritable and anxious temperament scores were inversely correlated. Blood pressure and irritable and anxious temperament scores were correlated. There was a strong correlation between waist circumference and cyclothymic and anxious temperament scores. There was no association between blood fasting glucose levels and affective temperament scores. However, there was a cluster of risk factors that include presence of MetS as well as a more negative prone temperament profile, that both incline patients to development of coronary heart disease and diabetes.

\section{Conclusion}

Consequently, there is a multidimensional description for $\mathrm{BD}$ 
that is coherent, comprehensive, and explanatory. Existence of MetS and the onset and progression of BD appear to be correlated. Previous depressive episode, seasonality, negative family history and childhood trauma are observed to be predictors of MetS. MetS (+) patients' anxious and irritable temperament scores were higher [3]. This connection may provide a thought-provoking new paradigm for studying the "systemic" nature of mood disorders. This may also contribute to the discovery of biological markers, growth in the number of our diagnostic tools, development of protective and individual-based options for treatment. At the moment, some endocrinological drugs may be influential in the treatment of mood disorders. Administering allopurinol and tamoxifen was found to be antimanic treatments in guidelines for the treatment of mood disorders $[5,63]$. Due to the fact that intranasal insulin is effective in treating cognitive impairment in $\mathrm{BD}$ and mood and cognitive symptoms in MDD, it should be recalled that the first ever treatment similar to electroconvulsive therapy was carried out by insulin shock [64]. Moreover, lithium and carbamazepine are known to directly effect terminal differentiation process and shorten the half-life of cancer cells in some leukemias and lymphomas [65]. Lithium and sodium valproate inhibit growth of carcinoid tumors, as well as reduce neuroendocrine tumor marker chromograninA levels [66].

\section{References}

1. Evans DL, Charney DS, Lewis L, Golden RN, Gorman JM, Krishnan KR, Nemeroff CB, et al. Mood disorders in the medically ill: scientific review and recommendations. Biol Psychiatry. 2005;58(3):175-189.

2. Fagiolini A, Goracci A. The effects of undertreated chronic medical illnesses in patients with severe mental disorders. J Clin Psychiatry. 2009;70(Suppl 3):22-29.

3. Kesebir S, Erdinc B, Tarhan N. Predictors of metabolic syndrome in first manic episode. Asian J Psychiatr. 2017;25:179-183.

4. Turan C, Kesebir S, Suner O. Are ICAM, VCAM and Eselectin levels different in first manic episode and subsequent remission? J Affect Disord. 2014;163:76-80.

5. Kesebir S, Tatlidil Yaylaci E, Suner O, Gultekin BK. Uric acid levels may be a biological marker for the differentiation of unipolar and bipolar disorder: the role of affective temperament. J Affect Disord. 2014;165:131-134.

6. Maina G, Bechon E, Rigardetto S, Salvi V. General medical conditions are associated with delay to treatment in patients with bipolar disorder. Psychosomatics. 2013;54(5):437-442.

7. Perugi G, Quaranta G, Belletti S, Casalini F, Mosti N, Toni C, Dell'Osso L. General medical conditions in 347 bipolar disorder patients: clinical correlates of metabolic and autoimmune-allergic diseases. J Affect Disord. 2015;170:95-103.

8. Margari F, Lozupone M, Pisani R, Pastore A, Todarello O, Zagaria G, Minerva F, et al. Metabolic syndrome: differences between psychiatric and internal medicine patients. Int J Psychiatry Med. 2013;45(3):203-226.
9. Kesebir S, Altintas M, Tatlidil Yaylaci E, Erdinc B, Tarhan N. Metabolic syndrome in first manic episode: A comparison between patients with or without previous depressive episode. J Metabolic Synd. 2016;5:201.

10. Gencer AG, Kesebir S. Diabetes in first episode mania: relations with clinical and the other endocrinological and metabolic parameters. Bipolar Disord. 2012;14:90.

11. Soreca I, Fagiolini A, Frank E, Houck PR, Thompson WK, Kupfer DJ. Relationship of general medical burden, duration of illness and age in patients with bipolar I disorder. J Psychiatr Res. 2008;42(11):956-961.

12. Kesebir S. Metabolic syndrome and childhood trauma: Also comorbidity and complication in mood disorder. World J Clin Cases. 2014;2(8):332-337.

13. Goldstein BI, Kemp DE, Soczynska JK, McIntyre RS. Inflammation and the phenomenology, pathophysiology, comorbidity, and treatment of bipolar disorder: a systematic review of the literature. J Clin Psychiatry. 2009;70(8):1078-1090.

14. Vancampfort D, Correll CU, Wampers M, Sienaert P, Mitchell AJ, De Herdt A, Probst M, et al. Metabolic syndrome and metabolic abnormalities in patients with major depressive disorder: a meta-analysis of prevalences and moderating variables. Psychol Med. 2014;44(10):20172028.

15. Luppino FS, Bouvy PF, Giltay EJ, Penninx BW, Zitman FG. The metabolic syndrome and related characteristics in major depression: inpatients and outpatients compared: metabolic differences across treatment settings. Gen Hosp Psychiatry. 2014;36(5):509-515.

16. Vancampfort D, Vansteelandt K, Correll CU, Mitchell AJ, De Herdt A, Sienaert P, Probst M, et al. Metabolic syndrome and metabolic abnormalities in bipolar disorder: a meta-analysis of prevalence rates and moderators. Am J Psychiatry. 2013;170(3):265-274.

17. Fitzgerald PJ. Is norepinephrine an etiological factor in some types of cancer? Int J Cancer. 2009;124(2):257263.

18. So HC, Gui AH, Cherny SS, Sham PC. Evaluating the heritability explained by known susceptibility variants: a survey of ten complex diseases. Genet Epidemiol. 2011;35(5):310-317.

19. Lee SH, Wray NR, Goddard ME, Visscher PM. Estimating missing heritability for disease from genome-wide association studies. Am J Hum Genet. 2011;88(3):294-305.

20. Kesebir S, Unubol B, Tatlidil Yaylaci E, Gundogar D, Unubol H. Impact of childhood trauma and affective temperament on resilience in bipolar disorder. Int J Bipolar Disord. 2015;3:3.

21. McIntyre RS, Soczynska JK, Liauw SS, Woldeyohannes HO, Brietzke E, Nathanson J, Alsuwaidan M, et al. The association between childhood adversity and components of metabolic syndrome in adults with mood disorders: results from the international mood disorders collaborative project. Int J Psychiatry Med. 2012;43(2):165-177.

22. McIntyre RS. Seasonal and temperamental contributions in patients with bipolar disorder and metabolic syndrome. Rev Bras Psiquiatr. 2013;35(2):210.

23. Kesebir S, Yasan Sair B, Unubol B, Tatlidil Yaylaci E. Is 
there a relationship between age at menarche and clinical and temperamental characteristics in bipolar disorder? Ann Clin Psychiatry. 2013;25(2):121-124.

24. Kesebir S, Guven S, Tatlidil Yaylaci E, Bilgin Topcuoglu O, Altintas M. EEG Abnormality in first episode mania: is it trait or state? Psychol Res. 2013;3:563-570.

25. Englund A, Kovanen L, Saarikoski ST, Haukka J, Reunanen A, Aromaa A, Lonnqvist J, et al. NPAS2 and PER2 are linked to risk factors of the metabolic syndrome. J Circadian Rhythms. 2009;7:5.

26. Rihmer Z, Purebl G, Faludi G, Halmy L. [Association of obesity and depression]. Neuropsychopharmacol Hung. 2008;10(4):183-189.

27. Kesebir S, Kucuksubasi Y, Tatlidil Yaylaci E. Metabolic syndrome in SAD: winter vs summer. Bipolar Disord. 2016;18:86.

28. Rintamaki R, Grimaldi S, Englund A, Haukka J, Partonen T, Reunanen A, Aromaa A, et al. Seasonal changes in mood and behavior are linked to metabolic syndrome. PLoS One. 2008;3(1):e1482.

29. Pan WH, Yeh WT, Weng LC. Epidemiology of metabolic syndrome in Asia. Asia Pac J Clin Nutr. 2008;17(Suppl 1):37-42.

30. Han L, Wang K, Cheng Y, Du Z, Rosenthal NE, Primeau F. Summer and winter patterns of seasonality in Chinese college students: a replication. Compr Psychiatry. 2000;41(1):57-62.

31. Levitan RD. The chronobiology and neurobiology of winter seasonal affective disorder. Dialogues Clin Neurosci. 2007;9(3):315-324.

32. Elbi H, Noyan A, Korukoglu S, Unal S, Bekaroglu M, Oguzhanoglu N, Turkoz N, et al. Seasonal affective disorder in eight groups in Turkey: a cross-national perspective. J Affect Disord. 2002;70(1):77-84.

33. Tonetti L, Sahu S, Natale V. Cross-national survey of winter and summer patterns of mood seasonality: a comparison between Italy and India. Compr Psychiatry. 2012;53(6):837-842.

34. Guzman A, Rohan KJ, Yousufi SM, Nguyen MC, Jackson MA, Soriano JJ, Postolache TT. Mood sensitivity to seasonal changes in African college students living in the greater Washington D.C. metropolitan area. ScientificWorldJournal. 2007; 7:584-591.

35. Magnusson A, Stefansson JG. Prevalence of seasonal affective disorder in Iceland. Arch Gen Psychiatry. 1993;50(12):941-946.

36. Grimaldi S, Englund A, Partonen T, Haukka J, Pirkola S, Reunanen A, Aromaa A, et al. Experienced poor lighting contributes to the seasonal fluctuations in weight and appetite that relate to the metabolic syndrome. J Environ Public Health. 2009;2009:165013.

37. Saheer TB, Lien L, Hauff E, Nirmal Kumar B. Ethnic differences in seasonal affective disorder and associated factors among five immigrant groups in Norway. J Affect Disord. 2013;151(1):237-242.

38. Madden PA, Heath AC, Rosenthal NE, Martin NG. Seasonal changes in mood and behavior. The role of genetic factors. Arch Gen Psychiatry. 1996;53(1):47-55.

39. Rosenthal NE, Genhart MJ, Caballero B, Jacobsen FM,
Skwerer RG, Coursey RD, Rogers S, et al. Psychobiological effects of carbohydrate- and protein-rich meals in patients with seasonal affective disorder and normal controls. Biol Psychiatry. 1989;25(8):1029-1040.

40. Lincoln GA, Clarke IJ, Hut RA, Hazlerigg DG. Characterizing a mammalian circannual pacemaker. Science. 2006;314(5807):1941-1944.

41. Stoleru D, Nawathean P, Fernandez MP, Menet JS, Ceriani MF, Rosbash M. The Drosophila circadian network is a seasonal timer. Cell. 2007;129(1):207-219.

42. Zambon AC, McDearmon EL, Salomonis N, Vranizan KM, Johansen KL, Adey D, Takahashi JS, et al. Timeand exercise-dependent gene regulation in human skeletal muscle. Genome Biol. 2003;4(10):R61.

43. Krauchi K, Cajochen C, Werth E, Wirz-Justice A. Alteration of internal circadian phase relationships after morning versus evening carbohydrate-rich meals in humans. J Biol Rhythms. 2002;17(4):364-376.

44. Gaist PA, Obarzanek E, Skwerer RG, Duncan CC, Shultz PM, Rosenthal NE. Effects of bright light on resting metabolic rate in patients with seasonal affective disorder and control subjects. Biol Psychiatry. 1990;28(11):989-996.

45. Kesebir S, Tatlidil Yaylaci E, Akdeniz F. Lithium induced hyperparathyroidism in lithium monotherapy: comparison of clinical properties of BD between before and after lithium monotherapy. Bipolar Disord. 2016;18:86.

46. Kesebir S, Tatlidil Yaylaci E, Akdeniz F. Vitamin D deficiency and hyperparathyroidism: a comparison between BD with comorbide ADHD and without. Bipolar Disord. 2016;18:80.

47. Bijlenga D, van der Heijden KB, Breuk M, van Someren EJ, Lie ME, Boonstra AM, Swaab HJ, et al. Associations between sleep characteristics, seasonal depressive symptoms, lifestyle, and ADHD symptoms in adults. J Atten Disord. 2013;17(3):261-275.

48. Lee HJ, Rex KM, Nievergelt CM, Kelsoe JR, Kripke DF. Delayed sleep phase syndrome is related to seasonal affective disorder. J Affect Disord. 2011;133(3):573-579.

49. Kesebir S. Projection of biphasic state to mitochondria. J Mol Histol Med Physiol. 2016;2:2.

50. Masri S, Zocchi L, Katada S, Mora E, Sassone-Corsi P. The circadian clock transcriptional complex: metabolic feedback intersects with epigenetic control. Ann N Y Acad Sci. 2012;1264:103-109.

51. Kesebir S, Vahip S, Akdeniz F, Yuncu Z, Alkan M, Akiskal H. Affective temperaments as measured by TEMPS$A$ in patients with bipolar I disorder and their first-degree relatives: a controlled study. J Affect Disord. 2005;85(12):127-133.

52. Altinbas K, Guloksuz S, Oral ET. Metabolic syndrome prevalence in different affective temperament profiles in bipolar-I disorder. Rev Bras Psiquiatr. 2013;35(2):131135.

53. Oginska H, Oginska-Bruchal K. Chronotype and personality factors of predisposition to seasonal affective disorder. Chronobiol Int. 2014;31(4):523-531.

54. Ravaja N, Keltikangas-Jarvinen L. Temperament and metabolic syndrome precursors in children: a three-year follow-up. Prev Med. 1995;24(5):518-527. 
55. Keltikangas-Jarvinen L, Ravaja N, Viikari J. Identifying Cloninger's temperament profiles as related to the early development of the metabolic cardiovascular syndrome in young men. Arterioscler Thromb Vasc Biol. 1999;19(8):1998-2006.

56. Mommersteeg PM, Pouwer F. Personality as a risk factor for the metabolic syndrome: a systematic review. J Psychosom Res. 2012;73(5):326-333.

57. Sovio U, King V, Miettunen J, Ek E, Laitinen J, Joukamaa M, Veijola J, et al. Cloninger's Temperament dimensions, socio-economic and lifestyle factors and metabolic syndrome markers at age 31 years in the Northern Finland Birth Cohort 1966. J Health Psychol. 2007;12(2):371382.

58. Tatlidil Yaylaci E, Kesebir S, Gungordu O. The relationship between impulsivity and lipid levels in bipolar patients: does temperament explain it? Compr Psychiatry. 2014;55(4):883-886.

59. Kesebir S, Tatlidil Yaylaci E, Demirkan AK, Altintas M. The relationship of impulsivity and lipid levels in bipolar patients: gender effect. Bipolar Disord. 2014;16:126.

60. Kesebir S, Tatlidil Yaylaci E, Atguden N, Altintas M. Metabolic parameters in first episode mania. Bipolar Disord. 2014;16:110.
61. Kesebir S, Vahip S, Akdeniz F, Yuncu Z. [The relationship of affective temperament and clinical features in bipolar disorder]. Turk Psikiyatri Derg. 2005;16(3):164-169.

62. Kesebir S, Erdinc B, Altintas M. Is there a relation between affective temperament and metabolic syndrome? Bipolar Disord. 2016;18:95.

63. Yildiz A, Guleryuz S, Ankerst DP, Ongur D, Renshaw PF. Protein kinase $\mathrm{C}$ inhibition in the treatment of mania: a double-blind, placebo-controlled trial of tamoxifen. Arch Gen Psychiatry. 2008;65(3):255-263.

64. Cha DS, Best MW, Bowie CR, Gallaugher LA, Woldeyohannes HO, Soczynska JK, Lewis G, et al. A randomized double-blind, placebo controlled, crossover trial evaluating the effect of intranasal insulin on cognition and mood in individuals with reatment resistant MDD. J Affect Disord. 2017;210:57-65.

65. Haffman FA, Kishter S, Nelson DL, Pizzo PA. Effect of lithium and other adjuvants on human $\mathrm{T}$ cell productionof, and response to interleukin II. Proc Am Assoc Cancer Res. 1981;22:280.

66. Adler JT, Hottinger DG, Kunnimalaiyaan M, Chen H. Combination therapy with histone deacetylase inhibitors and lithium chloride: a novel treatment for carcinoid tumors. Ann Surg Oncol. 2009;16(2):481-486. 